Acta Crystallographica Section E

Structure Reports

Online

ISSN 1600-5368

\section{2-[(3-Oxo-1-benzofuran-6-yl)oxy]aceto- nitrile}

\section{Henok H. Kinfe, Yonas H. Belay and Zanele H Phasha*}

Research Center for Synthesis and Catalysis, Department of Chemistry, University of Johannesburg (APK Campus), PO Box 524, Auckland Park, Johannesburg, 2006,

South Africa

Correspondence e-mail: zhphasha@uj.ac.za

Received 31 October 2012; accepted 26 November 2012

Key indicators: single-crystal X-ray study; $T=100 \mathrm{~K}$; mean $\sigma(\mathrm{C}-\mathrm{C})=0.002 \AA$; $R$ factor $=0.030 ; w R$ factor $=0.076 ;$ data-to-parameter ratio $=12.2$.

The molecule of the title compound, $\mathrm{C}_{11} \mathrm{H}_{8} \mathrm{O}_{3}$, is essentially planar [r.m.s. deviation $=0.025$ (2) $⿱$ A] . In the crystal, molecules are stacked along [110] but no short $\pi-\pi$ contacts are observed. Weak $\mathrm{C}-\mathrm{H} \cdots \mathrm{O}$ interactions link the molecules into chains along [101].

\section{Related literature}

For background to the development of hybrid drug candidates against tuberculosis, malaria and cancer, see: Morphy et al. (2004). For the synthesis of the title compound, see: Hoogendoorn et al. (2011).

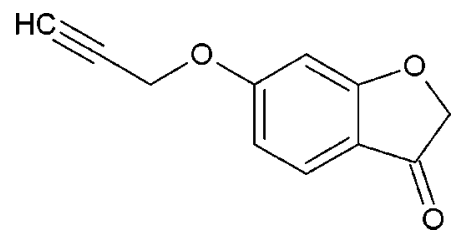

\section{Experimental}

Crystal data

$\mathrm{C}_{11} \mathrm{H}_{8} \mathrm{O}_{3}$

$M_{r}=188.17$

Monoclinic, $C 2 / c$

$$
\begin{aligned}
& a=16.8785(5) \AA \\
& b=5.4202(2) \AA \\
& c=19.6107(6) \AA
\end{aligned}
$$

$\beta=91.469(2)$

$V=1793.49(10) \AA^{3}$

$\mu=0.85 \mathrm{~mm}^{-1}$

$T=100 \mathrm{~K}$

$Z=8$

$0.19 \times 0.15 \times 0.11 \mathrm{~mm}$

$\mathrm{Cu} K \alpha$ radiation

Data collection

Bruker APEX DUO 4K CCD 10685 measured reflections diffractometer

Absorption correction: multi-scan (SADABS; Bruker, 2008)

$T_{\min }=0.855, T_{\max }=0.912$

Refinement

$R\left[F^{2}>2 \sigma\left(F^{2}\right)\right]=0.030$

$w R\left(F^{2}\right)=0.076$

$S=1.04$

1545 reflections

10685 measured reflections 1545 independent reflections 1451 reflections with $I>2 \sigma(I)$ $R_{\text {int }}=0.026$

127 parameters

$\mathrm{H}$-atom parameters constrained $\Delta \rho_{\max }=0.18 \mathrm{e} \AA^{-3}$

$\Delta \rho_{\min }=-0.13$ e $\AA^{-3}$

Table 1

Hydrogen-bond geometry $\left(\AA{ }^{\circ}\right)$.

\begin{tabular}{lllll}
\hline$D-\mathrm{H} \cdots A$ & $D-\mathrm{H}$ & $\mathrm{H} \cdots A$ & $D \cdots A$ & $D-\mathrm{H} \cdots A$ \\
\hline $\mathrm{C} 11-\mathrm{H} 11 \cdots \mathrm{O} 1^{\mathrm{i}}$ & 0.95 & 2.24 & $3.1676(15)$ & 165 \\
\hline Symmetry code: (i) $x-\frac{1}{2},-y+\frac{1}{2}, z-\frac{1}{2}$ & &
\end{tabular}

Data collection: APEX2 (Bruker, 2011); cell refinement: SAINT (Bruker, 2008); data reduction: SAINT and XPREP (Bruker, 2008); program(s) used to solve structure: SHELXS97 (Sheldrick, 2008); program(s) used to refine structure: SHELXL97 (Sheldrick, 2008); molecular graphics: ORTEP-3 for Windows (Farrugia, 2012); software used to prepare material for publication: WinGX (Farrugia, 2012).

Support by the research funds of the University of Johannesburg is gratefully acknowledged.

Supplementary data and figures for this paper are available from the IUCr electronic archives (Reference: LD2084).

\title{
References
}

Bruker (2008). SADABS, SAINT and XPREP. Bruker AXS Inc., Madison, Wisconsin, USA.

Bruker (2011). APEX2. Bruker AXS Inc., Madison, Wisconsin, USA.

Farrugia, L. J. (2012). J. Appl. Cryst. 45, 849-854.

Hoogendoorn, S., Blom, A. E. M., Willems, L. I., Van der Marel, G. A. \& Overkleeft, H. S. (2011). Org. Lett. 13, 5656-5659.

Morphy, R., Kay, C. \& Rankovic, Z. (2004). Drug Discov. Today, 9, 641-651. Sheldrick, G. M. (2008). Acta Cryst. A64, 112-122. 


\title{
supporting information
}

Acta Cryst. (2013). E69, o74 [https://doi.org/10.1107/S1600536812048441]

\section{2-[(3-Oxo-1-benzofuran-6-yl)oxy]acetonitrile}

\author{
Henok H. Kinfe, Yonas H. Belay and Zanele H Phasha
}

\section{S1. Comment}

As a continuation of our progress in the development of hybrid drug candidates against tuberculosis, malaria and cancer (Morphy et al., 2004), the title compound was identified as a promising starting material. The compound was synthesized by reaction of 6-Hydroxy-benzofuran-3-one with propargyl bromide at comparatively low temperature in the presence of potassium carbonate (Hoogendoorn et al., 2011). To confirm the effect of temperature on the reaction, herein we report the single-crystal structure of the title Compound.

The molecular structure of the compound is shown in Figure 1. The molecule is essentially planar (r.m.s. deviation = 0.025 (2) $\AA$ ). In the crystal the molecules are linked by infinite one-dimensional $\mathrm{C}-\mathrm{H} \cdots \mathrm{O}$ hydrogen bonding into chains that propagate in the [101] direction (Table 1, Figure 2).

\section{S2. Experimental}

A solution of 6-Hydroxy-benzofuran-3-one $(1 \mathrm{~g}, 6.66 \mathrm{~mm})$ in dry acetone was treated with potassium carbonate $(1.3 \mathrm{~g}$, $9.32 \mathrm{~mm}$ ). The reaction mixture was heated at a temperature of $40-50{ }^{\circ} \mathrm{C}$ for about 30 minutes and then propargyl bromide $(1.6 \mathrm{ml}, 14.65 \mathrm{~mm})$ was added to it. The combined solution was stirred for about $2.5 \mathrm{~h}$ and concentrated under vacuum. The residue was diluted with water and extracted three times with ethyl acetate. The combined organic layer was washed with brine and water and dried over anhydrous magnesium sulfate. After that filtered and the filtrate solid product was recrystalized from ethyl acetate and hexane to afford $80 \%$ of the target compound as yellow crystal.

Analytical data: m.p: 112 - $114 \mathrm{oC}$; ${ }^{1} \mathrm{H}$ NMR (CDCl3, $\left.400 \mathrm{MHZ}\right)$ : $\mathrm{d} 7.56$ (d, 1H), $6.69-6.64$ (m, 2H), 4.74 (s, 2H), 4.60 (s, 2H), 2.57(s, 1H); $\left.{ }^{13} \mathrm{C} \mathrm{NMR} \mathrm{(CDCl3,} 400 \mathrm{MHZ}\right)$ : d 197.6, 176.1, 165.8, 125.2, 115.0, 111.9, 97.7, 75.2, 56.2.

\section{S3. Refinement}

All hydrogen atoms were positioned in geometrically idealized positions with $\mathrm{C}-\mathrm{H}=0.99 \AA$ (methylene), $0.95 \AA$ (aromatic and acetylenic). All hydrogen atoms were allowed to ride on their parent atoms with $U_{\text {iso }}(\mathrm{H})=1.2 U_{\text {eq. }}$. The highest residual electron density of $0.18 \mathrm{e} . \AA^{-3}$ is $0.66 \AA$ from $\mathrm{C} 3$. 


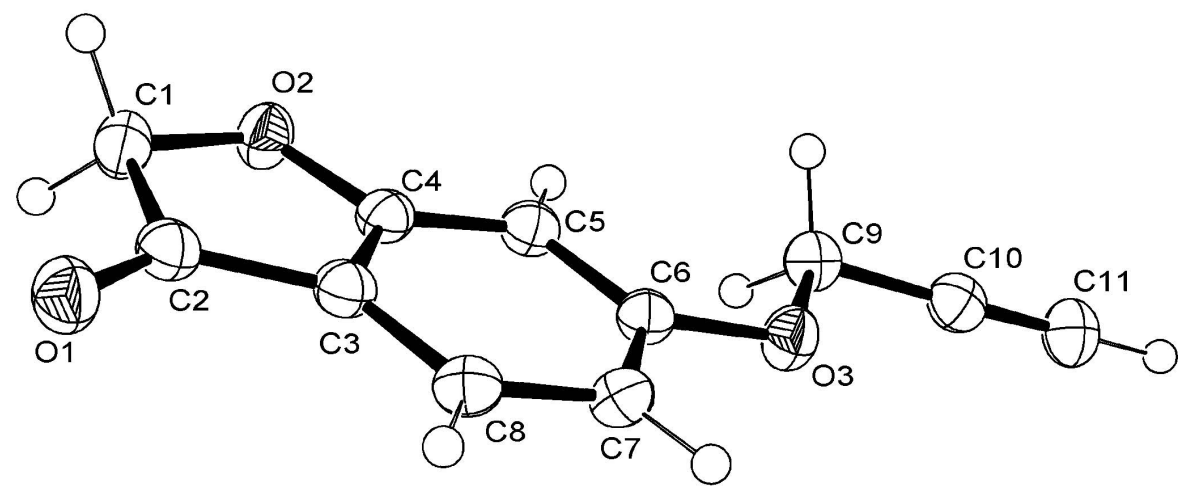

Figure 1

Crystal structure of the title compound with labeling and displacement ellipsoids drawn at the $50 \%$ probability level.

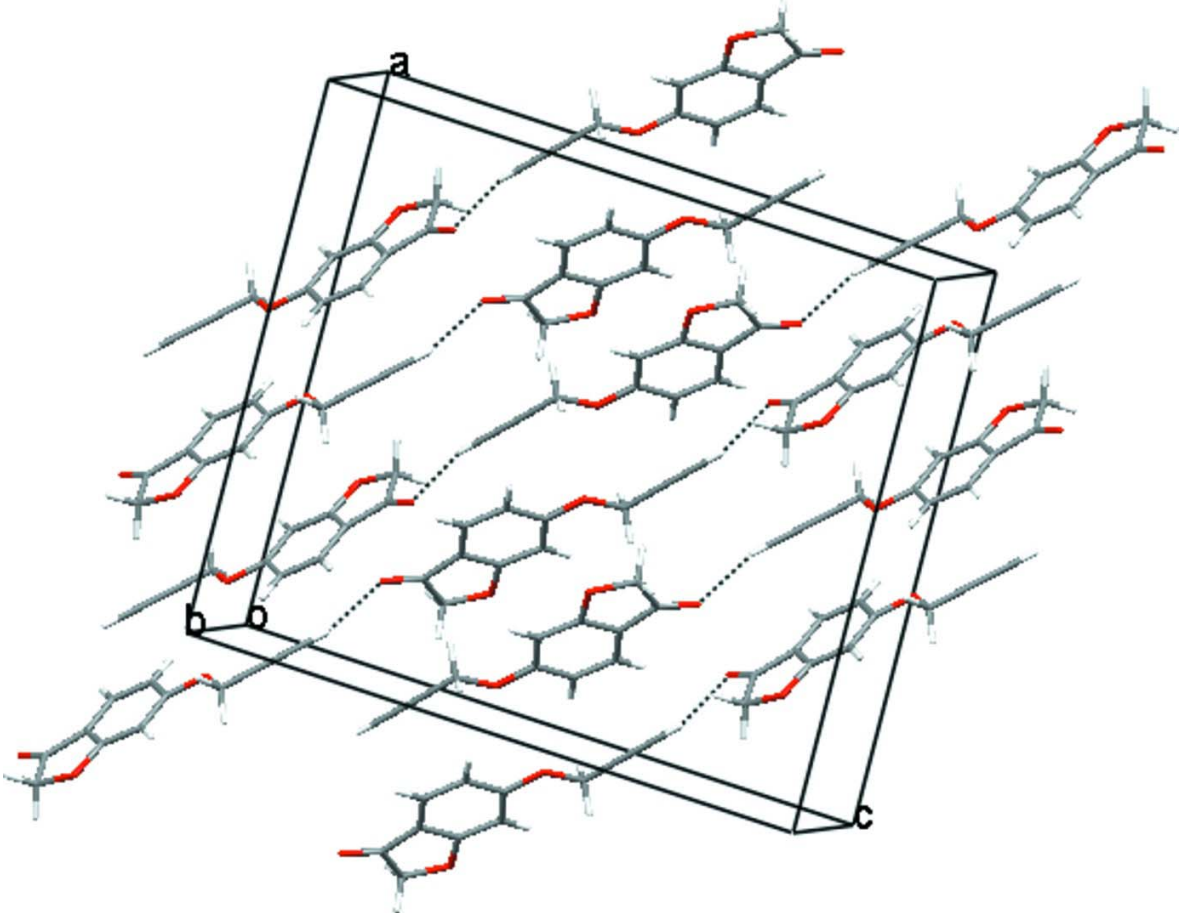

Figure 2

A portion of the crystal packing viewed approximately down the $b$ axis. Dotted lines show intermolecular $\mathrm{C}-\mathrm{H} \cdots \mathrm{O}$ interactions. 


\section{2-[(3-Oxo-1-benzofuran-6-yl)oxy]acetonitrile}

Crystal data

$\mathrm{C}_{11} \mathrm{H}_{8} \mathrm{O}_{3}$

$M_{r}=188.17$

Monoclinic, $C 2 / c$

Hall symbol: -C $2 \mathrm{yc}$

$a=16.8785(5) \AA$

$b=5.4202(2) \AA$

$c=19.6107(6) \AA$

$\beta=91.469(2)^{\circ}$

$V=1793.49(10) \AA^{3}$

$Z=8$

\section{Data collection}

Bruker APEX DUO 4K CCD diffractometer

Incoatec Quazar Multilayer Mirror monochromator

Detector resolution: 8.4 pixels $\mathrm{mm}^{-1}$

$\varphi$ and $\omega$ scans

Absorption correction: multi-scan

(SADABS; Bruker, 2008)

$T_{\min }=0.855, T_{\max }=0.912$

\section{Refinement}

Refinement on $F^{2}$

Least-squares matrix: full

$R\left[F^{2}>2 \sigma\left(F^{2}\right)\right]=0.030$

$w R\left(F^{2}\right)=0.076$

$S=1.04$

1545 reflections

127 parameters

0 restraints

Primary atom site location: structure-invariant direct methods
$F(000)=784$

$D_{\mathrm{x}}=1.394 \mathrm{Mg} \mathrm{m}^{-3}$

$\mathrm{Cu} K \alpha$ radiation, $\lambda=1.54178 \AA$

Cell parameters from 6173 reflections

$\theta=6.8-65.7^{\circ}$

$\mu=0.85 \mathrm{~mm}^{-1}$

$T=100 \mathrm{~K}$

Cube, yellow

$0.19 \times 0.15 \times 0.11 \mathrm{~mm}$

10685 measured reflections

1545 independent reflections

1451 reflections with $I>2 \sigma(I)$

$R_{\text {int }}=0.026$

$\theta_{\max }=66.2^{\circ}, \theta_{\min }=6.8^{\circ}$

$h=-19 \rightarrow 18$

$k=-6 \rightarrow 6$

$l=-22 \rightarrow 23$

\section{Special details}

Secondary atom site location: difference Fourier map

Hydrogen site location: inferred from neighbouring sites

$\mathrm{H}$-atom parameters constrained

$w=1 /\left[\sigma^{2}\left(F_{\mathrm{o}}^{2}\right)+(0.0326 P)^{2}+1.2605 P\right]$

where $P=\left(F_{\mathrm{o}}{ }^{2}+2 F_{\mathrm{c}}{ }^{2}\right) / 3$

$(\Delta / \sigma)_{\max }<0.001$

$\Delta \rho_{\max }=0.18$ e $\AA^{-3}$

$\Delta \rho_{\min }=-0.13$ e $\AA^{-3}$

Experimental. The intensity data was collected on a Bruker Apex DUO 4 K CCD diffractometer using an exposure time of $5 \mathrm{~s} /$ frame. A total of 2405 frames were collected with a frame width of $1^{\circ}$ covering up to $\theta=66.21^{\circ}$ with $98.0 \%$ completeness accomplished.

Geometry. All s.u.'s (except the s.u. in the dihedral angle between two 1.s. planes) are estimated using the full covariance matrix. The cell s.u.'s are taken into account individually in the estimation of s.u.'s in distances, angles and torsion angles; correlations between s.u.'s in cell parameters are only used when they are defined by crystal symmetry. An approximate (isotropic) treatment of cell s.u.'s is used for estimating s.u.'s involving l.s. planes.

Refinement. Refinement of $F^{2}$ against ALL reflections. The weighted $R$-factor $w R$ and goodness of fit $S$ are based on $F^{2}$, conventional $R$-factors $R$ are based on $F$, with $F$ set to zero for negative $F^{2}$. The threshold expression of $F^{2}>2 \sigma\left(F^{2}\right)$ is used only for calculating $R$-factors(gt) etc. and is not relevant to the choice of reflections for refinement. $R$-factors based on $F^{2}$ are statistically about twice as large as those based on $F$, and $R$ - factors based on ALL data will be even larger.

Fractional atomic coordinates and isotropic or equivalent isotropic displacement parameters $\left(\AA^{2}\right)$

\begin{tabular}{lllll}
\hline & $x$ & $y$ & $z$ & $U_{\text {iso }} * / U_{\text {eq }}$ \\
\hline $\mathrm{C} 1$ & $0.33402(7)$ & $-0.0134(2)$ & $0.16647(6)$ & $0.0301(3)$
\end{tabular}




$\begin{array}{lllll}\text { H1A } & 0.3908 & 0.0177 & 0.1582 & 0.036^{*} \\ \text { H1B } & 0.33 & -0.1366 & 0.2035 & 0.036^{*} \\ \text { C2 } & 0.29260(7) & 0.2247(2) & 0.18581(6) & 0.0275(3) \\ \text { C3 } & 0.22985(7) & 0.2558(2) & 0.13478(6) & 0.0258(3) \\ \text { C4 } & 0.23445(6) & 0.0603(2) & 0.08957(6) & 0.0252(3) \\ \text { C5 } & 0.18383(6) & 0.0313(2) & 0.03363(6) & 0.0256(3) \\ \text { H5 } & 0.188 & -0.1042 & 0.0032 & 0.031^{*} \\ \text { C6 } & 0.12641(6) & 0.2130(2) & 0.02471(6) & 0.0253(3) \\ \text { C7 } & 0.12021(7) & 0.4147(2) & 0.06963(6) & 0.0275(3) \\ \text { H7 } & 0.0802 & 0.5354 & 0.0618 & 0.033^{*} \\ \text { C8 } & 0.17159(7) & 0.4373(2) & 0.12444(6) & 0.0277(3) \\ \text { H8A } & 0.1678 & 0.5731 & 0.1548 & 0.033^{*} \\ \text { C9 } & 0.07331(7) & 0.0050(2) & -0.07344(6) & 0.0281(3) \\ \text { H9A } & 0.0623 & -0.1496 & -0.0485 & 0.034^{*} \\ \text { H9B } & 0.1263 & -0.009 & -0.0936 & 0.034^{*} \\ \text { C10 } & 0.01328(7) & 0.0443(2) & -0.12691(6) & 0.0295(3) \\ \text { C11 } & -0.03534(7) & 0.0700(2) & -0.17094(6) & 0.0340(3) \\ \text { H11 } & -0.0744 & 0.0906 & -0.2063 & 0.041^{*} \\ \text { O1 } & 0.31189(5) & 0.35618(16) & 0.23405(4) & 0.0328(2) \\ \text { O2 } & 0.29422(5) & -0.10199(15) & 0.10488(4) & 0.0296(2) \\ \text { O3 } & 0.07151(5) & 0.21196(15) & -0.02749(4) & 0.0289(2)\end{array}$

Atomic displacement parameters $\left(\AA^{2}\right)$

\begin{tabular}{lllllll}
\hline & $U^{11}$ & $U^{22}$ & $U^{33}$ & $U^{12}$ & $U^{13}$ & $U^{23}$ \\
\hline C1 & $0.0269(6)$ & $0.0336(7)$ & $0.0295(6)$ & $0.0015(5)$ & $-0.0041(5)$ & $0.0012(5)$ \\
C2 & $0.0270(6)$ & $0.0289(6)$ & $0.0265(6)$ & $-0.0038(5)$ & $0.0012(5)$ & $0.0022(5)$ \\
C3 & $0.0264(6)$ & $0.0253(6)$ & $0.0257(6)$ & $-0.0028(5)$ & $0.0015(4)$ & $0.0019(5)$ \\
C4 & $0.0226(5)$ & $0.0245(6)$ & $0.0285(6)$ & $0.0000(4)$ & $0.0032(4)$ & $0.0043(5)$ \\
C5 & $0.0262(6)$ & $0.0246(6)$ & $0.0261(6)$ & $-0.0008(5)$ & $0.0029(4)$ & $-0.0001(5)$ \\
C6 & $0.0243(6)$ & $0.0269(6)$ & $0.0248(6)$ & $-0.0023(5)$ & $0.0010(4)$ & $0.0040(5)$ \\
C7 & $0.0288(6)$ & $0.0236(6)$ & $0.0303(6)$ & $0.0022(5)$ & $-0.0001(5)$ & $0.0025(5)$ \\
C8 & $0.0313(6)$ & $0.0232(6)$ & $0.0288(6)$ & $-0.0003(5)$ & $0.0018(5)$ & $-0.0001(5)$ \\
C9 & $0.0276(6)$ & $0.0295(6)$ & $0.0273(6)$ & $0.0010(5)$ & $0.0016(5)$ & $-0.0016(5)$ \\
C10 & $0.0279(6)$ & $0.0311(6)$ & $0.0296(6)$ & $-0.0003(5)$ & $0.0047(5)$ & $-0.0010(5)$ \\
C11 & $0.0297(6)$ & $0.0418(7)$ & $0.0304(6)$ & $0.0012(5)$ & $-0.0018(5)$ & $-0.0021(5)$ \\
O1 & $0.0324(5)$ & $0.0349(5)$ & $0.0307(5)$ & $-0.0012(4)$ & $-0.0048(3)$ & $-0.0026(4)$ \\
O2 & $0.0273(4)$ & $0.0297(5)$ & $0.0316(4)$ & $0.0052(3)$ & $-0.0036(3)$ & $-0.0019(3)$ \\
O3 & $0.0288(4)$ & $0.0298(4)$ & $0.0278(4)$ & $0.0034(3)$ & $-0.0046(3)$ & $-0.0026(3)$ \\
& & & & & &
\end{tabular}

Geometric parameters $\left(\AA,{ }^{o}\right)$

\begin{tabular}{llll}
\hline $\mathrm{C} 1-\mathrm{O} 2$ & $1.4489(14)$ & $\mathrm{C} 6-\mathrm{O} 3$ & $1.3631(13)$ \\
$\mathrm{C} 1-\mathrm{C} 2$ & $1.5206(17)$ & $\mathrm{C} 6-\mathrm{C} 7$ & $1.4095(16)$ \\
$\mathrm{C} 1-\mathrm{H} 1 \mathrm{~A}$ & 0.99 & $\mathrm{C} 7-\mathrm{C} 8$ & $1.3691(17)$ \\
$\mathrm{C} 1-\mathrm{H} 1 \mathrm{~B}$ & 0.99 & $\mathrm{C} 7-\mathrm{H} 7$ & 0.95 \\
$\mathrm{C} 2-\mathrm{O} 1$ & $1.2220(14)$ & $\mathrm{C} 8-\mathrm{H} 8 \mathrm{~A}$ & 0.95 \\
$\mathrm{C} 2-\mathrm{C} 3$ & $1.4481(16)$ & $\mathrm{C} 9-\mathrm{O} 3$ & $1.4396(14)$
\end{tabular}




\begin{tabular}{|c|c|c|c|}
\hline $\mathrm{C} 3-\mathrm{C} 4$ & $1.3851(16)$ & $\mathrm{C} 9-\mathrm{C} 10$ & $1.4551(16)$ \\
\hline $\mathrm{C} 3-\mathrm{C} 8$ & $1.4021(17)$ & C9-H9A & 0.99 \\
\hline $\mathrm{C} 4-\mathrm{O} 2$ & $1.3661(14)$ & C9-H9B & 0.99 \\
\hline $\mathrm{C} 4-\mathrm{C} 5$ & $1.3822(16)$ & $\mathrm{C} 10-\mathrm{C} 11$ & $1.1840(17)$ \\
\hline $\mathrm{C} 5-\mathrm{C} 6$ & $1.3896(16)$ & C11-H11 & 0.95 \\
\hline $\mathrm{C} 5-\mathrm{H} 5$ & 0.95 & & \\
\hline $\mathrm{O} 2-\mathrm{C} 1-\mathrm{C} 2$ & $106.42(9)$ & $\mathrm{O} 3-\mathrm{C} 6-\mathrm{C} 7$ & $114.37(10)$ \\
\hline $\mathrm{O} 2-\mathrm{C} 1-\mathrm{H} 1 \mathrm{~A}$ & 110.4 & $\mathrm{C} 5-\mathrm{C} 6-\mathrm{C} 7$ & $122.25(10)$ \\
\hline $\mathrm{C} 2-\mathrm{C} 1-\mathrm{H} 1 \mathrm{~A}$ & 110.4 & $\mathrm{C} 8-\mathrm{C} 7-\mathrm{C} 6$ & $120.30(11)$ \\
\hline $\mathrm{O} 2-\mathrm{C} 1-\mathrm{H} 1 \mathrm{~B}$ & 110.4 & $\mathrm{C} 8-\mathrm{C} 7-\mathrm{H} 7$ & 119.8 \\
\hline $\mathrm{C} 2-\mathrm{C} 1-\mathrm{H} 1 \mathrm{~B}$ & 110.4 & $\mathrm{C} 6-\mathrm{C} 7-\mathrm{H} 7$ & 119.8 \\
\hline $\mathrm{H} 1 \mathrm{~A}-\mathrm{C} 1-\mathrm{H} 1 \mathrm{~B}$ & 108.6 & $\mathrm{C} 7-\mathrm{C} 8-\mathrm{C} 3$ & $118.58(11)$ \\
\hline $\mathrm{O} 1-\mathrm{C} 2-\mathrm{C} 3$ & $129.99(11)$ & $\mathrm{C} 7-\mathrm{C} 8-\mathrm{H} 8 \mathrm{~A}$ & 120.7 \\
\hline $\mathrm{O} 1-\mathrm{C} 2-\mathrm{C} 1$ & $124.96(11)$ & $\mathrm{C} 3-\mathrm{C} 8-\mathrm{H} 8 \mathrm{~A}$ & 120.7 \\
\hline $\mathrm{C} 3-\mathrm{C} 2-\mathrm{C} 1$ & $105.04(9)$ & $\mathrm{O} 3-\mathrm{C} 9-\mathrm{C} 10$ & $108.15(9)$ \\
\hline $\mathrm{C} 4-\mathrm{C} 3-\mathrm{C} 8$ & $119.66(10)$ & $\mathrm{O} 3-\mathrm{C} 9-\mathrm{H} 9 \mathrm{~A}$ & 110.1 \\
\hline $\mathrm{C} 4-\mathrm{C} 3-\mathrm{C} 2$ & $107.53(10)$ & $\mathrm{C} 10-\mathrm{C} 9-\mathrm{H} 9 \mathrm{~A}$ & 110.1 \\
\hline $\mathrm{C} 8-\mathrm{C} 3-\mathrm{C} 2$ & $132.81(11)$ & $\mathrm{O} 3-\mathrm{C} 9-\mathrm{H} 9 \mathrm{~B}$ & 110.1 \\
\hline $\mathrm{O} 2-\mathrm{C} 4-\mathrm{C} 5$ & $122.62(10)$ & $\mathrm{C} 10-\mathrm{C} 9-\mathrm{H} 9 \mathrm{~B}$ & 110.1 \\
\hline $\mathrm{O} 2-\mathrm{C} 4-\mathrm{C} 3$ & $113.91(10)$ & $\mathrm{H} 9 \mathrm{~A}-\mathrm{C} 9-\mathrm{H} 9 \mathrm{~B}$ & 108.4 \\
\hline $\mathrm{C} 5-\mathrm{C} 4-\mathrm{C} 3$ & $123.47(11)$ & $\mathrm{C} 11-\mathrm{C} 10-\mathrm{C} 9$ & $178.27(13)$ \\
\hline $\mathrm{C} 4-\mathrm{C} 5-\mathrm{C} 6$ & $115.74(11)$ & $\mathrm{C} 10-\mathrm{C} 11-\mathrm{H} 11$ & 180 \\
\hline $\mathrm{C} 4-\mathrm{C} 5-\mathrm{H} 5$ & 122.1 & $\mathrm{C} 4-\mathrm{O} 2-\mathrm{C} 1$ & $107.09(9)$ \\
\hline $\mathrm{C} 6-\mathrm{C} 5-\mathrm{H} 5$ & 122.1 & $\mathrm{C} 6-\mathrm{O} 3-\mathrm{C} 9$ & $116.64(9)$ \\
\hline $\mathrm{O} 3-\mathrm{C} 6-\mathrm{C} 5$ & $123.37(10)$ & & \\
\hline $\mathrm{O} 2-\mathrm{C} 1-\mathrm{C} 2-\mathrm{O} 1$ & $-177.45(10)$ & $\mathrm{C} 4-\mathrm{C} 5-\mathrm{C} 6-\mathrm{C} 7$ & $0.20(16)$ \\
\hline $\mathrm{O} 2-\mathrm{C} 1-\mathrm{C} 2-\mathrm{C} 3$ & $1.34(12)$ & $\mathrm{O} 3-\mathrm{C} 6-\mathrm{C} 7-\mathrm{C} 8$ & $179.74(10)$ \\
\hline $\mathrm{O} 1-\mathrm{C} 2-\mathrm{C} 3-\mathrm{C} 4$ & $177.78(12)$ & $\mathrm{C} 5-\mathrm{C} 6-\mathrm{C} 7-\mathrm{C} 8$ & $-0.07(17)$ \\
\hline $\mathrm{C} 1-\mathrm{C} 2-\mathrm{C} 3-\mathrm{C} 4$ & $-0.93(12)$ & $\mathrm{C} 6-\mathrm{C} 7-\mathrm{C} 8-\mathrm{C} 3$ & $-0.23(17)$ \\
\hline $\mathrm{O} 1-\mathrm{C} 2-\mathrm{C} 3-\mathrm{C} 8$ & $-1.2(2)$ & $\mathrm{C} 4-\mathrm{C} 3-\mathrm{C} 8-\mathrm{C} 7$ & $0.40(16)$ \\
\hline $\mathrm{C} 1-\mathrm{C} 2-\mathrm{C} 3-\mathrm{C} 8$ & $-179.91(12)$ & $\mathrm{C} 2-\mathrm{C} 3-\mathrm{C} 8-\mathrm{C} 7$ & $179.28(11)$ \\
\hline $\mathrm{C} 8-\mathrm{C} 3-\mathrm{C} 4-\mathrm{O} 2$ & $179.30(10)$ & $\mathrm{C} 5-\mathrm{C} 4-\mathrm{O} 2-\mathrm{C} 1$ & $-179.70(10)$ \\
\hline $\mathrm{C} 2-\mathrm{C} 3-\mathrm{C} 4-\mathrm{O} 2$ & $0.16(13)$ & $\mathrm{C} 3-\mathrm{C} 4-\mathrm{O} 2-\mathrm{C} 1$ & $0.73(12)$ \\
\hline $\mathrm{C} 8-\mathrm{C} 3-\mathrm{C} 4-\mathrm{C} 5$ & $-0.27(17)$ & $\mathrm{C} 2-\mathrm{C} 1-\mathrm{O} 2-\mathrm{C} 4$ & $-1.26(12)$ \\
\hline $\mathrm{C} 2-\mathrm{C} 3-\mathrm{C} 4-\mathrm{C} 5$ & $-179.41(10)$ & $\mathrm{C} 5-\mathrm{C} 6-\mathrm{O} 3-\mathrm{C} 9$ & $2.07(15)$ \\
\hline $\mathrm{O} 2-\mathrm{C} 4-\mathrm{C} 5-\mathrm{C} 6$ & $-179.56(10)$ & $\mathrm{C} 7-\mathrm{C} 6-\mathrm{O} 3-\mathrm{C} 9$ & $-177.74(9)$ \\
\hline $\mathrm{C} 3-\mathrm{C} 4-\mathrm{C} 5-\mathrm{C} 6$ & $-0.03(16)$ & $\mathrm{C} 10-\mathrm{C} 9-\mathrm{O} 3-\mathrm{C} 6$ & $-177.25(9)$ \\
\hline $\mathrm{C} 4-\mathrm{C} 5-\mathrm{C} 6-\mathrm{O} 3$ & $-179.59(10)$ & & \\
\hline
\end{tabular}

Hydrogen-bond geometry $\left(\AA,{ }^{\circ}\right)$

\begin{tabular}{lllll}
\hline$D-\mathrm{H} \cdots A$ & $D-\mathrm{H}$ & $\mathrm{H} \cdots A$ & $D \cdots A$ & $D-\mathrm{H} \cdots A$ \\
\hline $\mathrm{C} 11-\mathrm{H} 11 \cdots \mathrm{O}^{\mathrm{i}}$ & 0.95 & 2.24 & $3.1676(15)$ & 165 \\
\hline
\end{tabular}

Symmetry code: (i) $x-1 / 2,-y+1 / 2, z-1 / 2$. 